Research Article

\title{
Local Cultural IP Development and Cultural Creative Design Based on Big Data and Internet of Things
}

\author{
Wei $\mathrm{Bi}^{1}$ and Guangming Wang $\mathbb{D}^{2}$ \\ ${ }^{1}$ Art and Design College, Guangdong University of Finance and Economics, Guangzhou 510320, Guangdong, China \\ ${ }^{2}$ School of Politics and Public Administration, Zhengzhou University, Zhengzhou 450001, Henan, China \\ Correspondence should be addressed to Guangming Wang; zzdxkjb@gs.zzu.edu.cn
}

Received 2 March 2021; Revised 24 March 2021; Accepted 8 April 2021; Published 20 April 2021

Academic Editor: Mian Ahmad Jan

Copyright (c) 2021 Wei Bi and Guangming Wang. This is an open access article distributed under the Creative Commons Attribution License, which permits unrestricted use, distribution, and reproduction in any medium, provided the original work is properly cited.

\begin{abstract}
Relying on the development of cultural tourism resources and the development of cultural tourism industry to achieve regional industrial revitalization is an important way of implementing postdisaster reconstruction in areas suffering from major natural disasters. To this end, this article proposes a local cultural IP development and cultural creative design method based on big data and the Internet of Things to explore new ideas for postdisaster reconstruction in such areas. First, we collect traditional and modern cultural element data and carry out data cleaning and processing through the Internet of Things. Second, we use data mining to perform multilayer collaborative processing on regional cultural data based on ontology modeling and tensor decomposition. Based on our approach, local cultural categories can be effectively screened and filtered out. Finally, we establish a cultural IP development model based on the Internet of Things and verify the validity and applicability of the model through system testing and simulation analysis.
\end{abstract}

\section{Introduction}

The deep integration and development of cultural tourism have become a major national strategy in the new era [1]. Culture is the soul of tourism, and tourism is an important carrier of culture. From the perspective of theory and industrial practice of culture and tourism research, the simple development and utilization of cultural resources and tourism resources are no longer adapted to the current development trend of the world's cultural tourism industry [2]. Since the 1970s, the in-depth involvement of multiple disciplines and industries represented by management, culture, history, geography, sociology, political science, and economics has made significant development in cultural and tourism industry $[3,4]$. Since the 21 st century, "promoting tourism with culture, excelling culture with tourism, and integrating culture and tourism" has become an important trend in the development of global cultural tourism industry [5].

It is important to effectively coordinate the development of national cultural undertakings, cultural industries, and tourism and effectively improve the country's cultural soft power and influence $[6,7]$. The research on cultural tourism resources in western academia mainly focuses on the constituent elements of cultural tourism resources and their utilization and development. In the aspect of research on the constituent elements of cultural tourism resources, Robert Dodo explained the relationship between the elements of Ashanti cultural resources and the perception and utilization of resources from the perspectives of cultural geography and cultural anthropology [8]. Thomas F King proposed that the evaluation of influence elements of American "cultural resources" should not be limited to archaeological sites or historical buildings, but more widely, effective methods should be used to use more diverse evaluation dimensions [9]. These methods should be used while determining whether a project is implemented or not by considering its impact on cultural resources in accordance with relevant laws and regulations [10]. With the continuous increase in people's leisure time and the vigorous development of tourism, the integration of cultural industry and the tourism 
industry continued to deepen. Some scholars have put forward their own views on this deepening of ties between these two industries. Wiener [11] compared and observed the development trends of the cultural industry and tourism in the United States and pointed out that cultural tourism's resources are a key element in strengthening the cooperation between these two industries to cultivate mutually beneficial new markets. Relying on the development of cultural tourism resources and the development of cultural tourism industry to achieve regional industrial revitalization is one of the important ways to implement postdisaster reconstruction in areas suffering from major natural disasters [12]. The postdisaster reconstruction in different regions of the world contains government practices in the development of local cultural tourism resources. More and more regions suffering from natural disasters are developing the cultural tourism industry by designing local cultural tourism resources to revitalize the local economy, politics, society, and culture $[13,14]$. As a whole, the rational development of local cultural tourism resources can produce better economic and social benefits to the disaster-stricken areas.

For this purpose, governments at all levels mobilize the available forces by performing their own functions to guide and promote the rational and efficient development of local cultural tourism resources and the healthy development of cultural tourism industry from different perspectives [15]. The major contributions of this paper are as follows:

(i) We propose a local cultural IP development and cultural creative design method based on big data and the Internet of Things to explore new ideas for postdisaster reconstruction in areas suffering from natural disasters.

(ii) We evaluated our work comprehensively by performing experimental results. These results justify the validity and efficiency of our work.

The rest of this paper is organized as follows. In Section 2 , we discuss an overview of related research on the Internet of Things and local cultural IP development. In Section 3, we discuss the classification and selection process of local cultural data based on data mining. In Section 4, we discuss the applicability of the model and the comparative testing process. Finally, we discuss the limitations of this article and future research prospects in Section 5.

\section{Related Search}

In this section, first we provide a comprehensive literature overview on the Internet of Things followed by local cultural IP development. The presentation of literature review on Internet of Things lays a solid basis for the justification of using it for local cultural IP development.

2.1. Internet of Things Technology. The Internet of Things (IoT) edges the gap between real-world and virtual world by connecting physical world devices with the Internet. For example, connecting radio, television, microwave, and similar devices with the Internet enable all ordinary devices to exchange their data $[16,17]$. The Internet of Things is generally a wireless network, but because everyone is around, there can be as many as one thousand to five thousand Internet of Things devices, so the Internet of Things system may contain 500 to 1,000 trillion Internet of Things devices. In the Internet of Things system, everyone can use electronic tags to connect real objects to the network, and in the Internet of Things system, owners can find out their specific locations. Central computers for the Internet of Things can be used to centrally manage and control equipment and personnel and can also remotely control related equipment in the home and can also perform location search and item antitheft and so on, similar to an automated operating system, and less through mobile phones, the data can be integrated into big data to realize the connection between objects and objects [18, 19]. In the perception layer, if we need to obtain information about items in the Internet of Things system, we need to read the relevant tags attached to the items through a reader. The privacy of tag data will lead to the leakage of key information of the entire network which has a devastating impact. Therefore, label privacy is one of the important objects for privacy protection in the Internet of Things [20]. Privacy includes information privacy and location privacy. Information privacy means that an attacker cannot obtain private information such as the identity of the tag and the reader and other information related to the user of the tag or the reader [21].

2.2. Local Cultural IP Development. In a broad sense, cultural tourism as an activity experience for people has existed in ancient times. Since the 1980s, cultural tourism has become an important part of the international tourism market $[22,23]$. From the perspective of the resource itself, according to the interpretation of the "Modern Chinese Dictionary," resource is a natural source of production and living materials and is the basis for human survival and social development. Marx also pointed out that "Labor and land are the two primitive forming elements of wealth." Engels believed that the creation of wealth requires materials provided by nature and the transformation of human labor, both of which are indispensable. It can be seen that resources refer to substances or materials formed in nature that can be used for human survival and social development. From the perspective of application field, cultural tourism resources, as the name suggests, are the resources used in the cultural tourism field, which can be regarded as natural, cultural, and social resources used in the "cultural creation" behavior of the tourism business main body [24, 25].

From the perspective of resource attributes, cultural tourism resources have the dual nature of both cultural resources and tourism resources [26, 27]. From the perspective of cultural resources, cultural resources include various resources that people can use in cultural production or activities. Cultural tourism activities from the subject "cultural creation" to the object "cultural consumption" all contain the characteristics of cultural resources. The difference between cultural tourism resources and cultural 
resources lies in the application of the subject of "cultural production" in the tourism field. From the perspective of tourism resources, foreign countries call tourism resources tourism attractions, that is, "any natural, humanistic object, or other factors that can attract tourists to generate tourism motives and may be used to develop tourism activities can be called tourism attractions [28]. Therefore, the difference between cultural tourism resources and tourism resources is that they can satisfy tourists' pursuit of cultural knowledge and spiritual level to a greater extent and can bring certain economic, social, and cultural benefits to tourist destinations [29]. The "postdisaster" discussed in this article is a broad concept. After the occurrence of natural disasters in the sense of time and space, the impact of natural disasters on Beichuan society, economy, and culture is the state to be reconstructed as shown by the industrial foundation, local culture, and residents' social psychology. Based on this, this article intends to analyze and discuss the development of local cultural IP and the design of cultural creation from the perspective of the development of local cultural tourism resources after the disaster [30].

\section{Classification and Screening Process of Local Cultural Data Based on Data Mining}

In the process of using machine learning methods to classify cultural data, data preprocessing is an important preprocessing stage, which has an important impact on the back-end classification results. Data preprocessing can transform the initial problem from high-dimensional space to low-dimensional space, thereby reducing computational complexity and improving classification efficiency. Correspondingly, it can eliminate irrelevant or redundant features, prevent the classifier from overfitting, and enhance the quality of the classification algorithm [31].

MultiSURF is an algorithm based on Relief. It is an algorithm that judges the importance of elements by weighting them. Each feature is assigned a different weight according to the degree of association between each attribute and class label, where the weight is less than the setting the elements of the threshold value which will be deleted. MultiSURF pseudocode is given in Figure 1. First, the operator randomly selects a sample of $R$, from the training set, and then searches for nearest neighbor samples of the same category as $R$ and nearest neighbor samples of different categories from $R$ [32]. It is worth noting that MultiSURF uses the boundary value Tear to determine which instances will be regarded as neighbors, $R$ is the average pairwise distance between other instances, and $a$ is the cost between the target instance $R$ and all other instances. The pseudocode flow chart of the MultiSURF algorithm is shown in Figure 2.

The MultiSURF algorithm is very efficient and does not interfere with the data type. It assigns different weights based on the performance of a single feature in the calculation phase to identify the category and then uses the set weight threshold to select a feature subset:

$$
g^{i}(x, y)=\lambda^{\prime} f^{i}(x, y) .
$$

However, in practical problems, a single feature usually cannot effectively classify categories. Correspondingly, the algorithm does not consider the correlation between features. Therefore, the downside of the algorithm is that it cannot eliminate redundant features well.

$$
E=E\left(y_{1}^{l}, \ldots, y_{n}^{l}\right)=\sum_{j}^{h}\left(y_{j}^{l}-t_{j}\right) .
$$

Since redundant features do not increase data information, they can affect the confidence of model classification, and the effect of redundant features will also be superimposed on the model, making the model effect worse.

Genetic algorithm is one of the most advanced algorithms for classification and screening. It is a type of the search heuristic method, inspired by Charles Darwin's theory of natural evolution, reflecting the process of natural selection. The process of natural selection is to select the most suitable individual from the population. The offspring continuously produced in this process will inherit some of the characteristics of the parents and add some characteristics to the next generation.

$$
\begin{aligned}
F\left(X_{n+1} \mid J_{n}\right) & \neq F\left(X_{n+1} \mid J_{n}-Y_{n}\right), \\
\lambda & =\max \frac{1}{N M}\left\{\sum_{k=0}^{L-1} f_{k}^{r}(x, y), \sum_{k=0}^{L-1} f_{k}^{d}(x, y), \sum_{k=0}^{L-1} f_{k}^{b}(x, y)\right\} .
\end{aligned}
$$

If parents have better adaptability, their offspring may be stronger than their parents and have a better chance of survival. This process will be repeated continuously, and finally, the most suitable generation will be found. In this process, as in the case of biological evolution, it has a mechanism to select suitable chromosomes in each generation. In order to simulate the evolutionary process, the selected chromosomes undergo genetic operations, such as crossover and mutation, and finally select the most suitable individuals for reproduction. In order to produce the next generation of offspring, this concept can be used to search for problems, that is, to prepare a set of solutions to the problem and select the best solution from it.

$$
\Delta f_{r}=\left[\frac{\partial f_{r}}{\partial x_{1}}, \frac{\partial f_{r}}{\partial x_{2}}, \ldots, \frac{\partial f_{r}}{\partial x_{n}}\right] .
$$

The fitness function determines the survivability of an individual (the ability of an individual to compete with other individuals). Each individual will get a fitness value through calculation, and the probability of an individual being selected for reproduction is based on its fitness score. The main purpose of the selection phase is to select the most suitable individuals and let them pass their genes to the next generation. Figure 1 shows the algorithm flow of the local cultural IP development model based on data mining.

A pair of individuals (parents) is selected based on their fitness scores. Individuals with higher scores have more chances to be selected for reproduction. Crossover is the most important stage in the genetic algorithm. For each pair of parents to mate, an exchange point needs to be randomly 


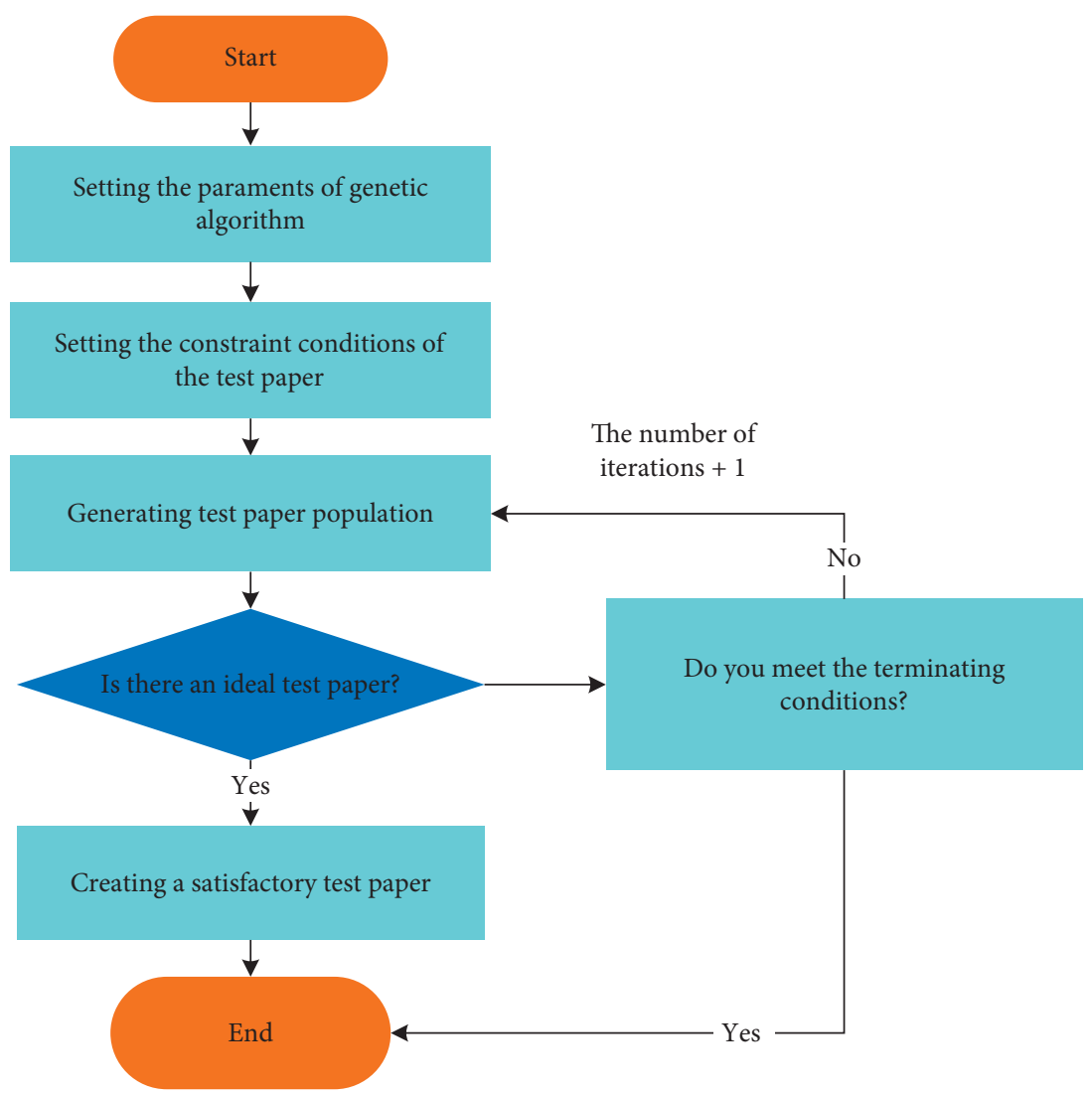

Figure 1: Algorithmic flow of the local cultural IP development model based on data mining.

selected from the genes for exchange. Offspring are created by exchanging parental genes until the exchange point is reached, and then new offspring are added to the population.

$$
E\left(X_{n+1} \mid J_{n}\right) \neq E\left(X_{n+1} \mid J_{n}-Y_{n}\right) \text {. }
$$

In some of the new offspring formed, some of their genes may be mutated with low random probability. This means that certain bits in the chromosome can be flipped, and the main purpose of mutation is to maintain the diversity within the population and prevent premature fusion. As the algorithm continues to repeat, if the population has converged (the algorithm does not produce offspring that are significantly different from the previous generation), the algorithm terminates.

$$
X_{t}=\sum_{i=1}^{k} \alpha_{i} X_{t-i}+\sum_{i=1}^{k} \beta_{i} Y_{t-i}+u_{1 t}
$$

As a search heuristic algorithm for solving optimization, the genetic algorithm is one of evolutionary algorithms. When the number of iterations of GA continues to increase, the overall fitness value of each generation will gradually converge to the local optimal solution, so the selected optimal feature subset can be obtained. In addition, GA usually uses the accuracy of the classification learning algorithm as a fitness function.

$$
\begin{aligned}
x^{(j+1)} & =x^{(j)}+s_{j} d^{(j)}, \\
\left|f^{(j)}-f^{(j-1)}\right| & \leq \tau, \\
\left|f^{(j)}-f^{(b)}\right| & \leq \tau, \\
F & =\frac{\left(\mathrm{SSE}_{r}-\mathrm{SSE}_{u}\right) / k}{\operatorname{SSE}_{u} /(T-2 k)} \sim F_{(k, T-2 k)} .
\end{aligned}
$$

In order to facilitate subsequent experiments to compare the pros and cons of the three different classifications in the screening algorithm and to verify the performance of the MSGA feature selection algorithm proposed in this paper, we used the three algorithms to select their respective feature subsets, of which the MultiSURF method selected 8 Features, 10 features were selected based on the GA-wrapped method, and 8 features were selected by MSGA. The 8 features selected using the MultiSURF feature selection algorithm are shown in Table 1.

We use the MultiSURF algorithm to sort all the features and delete some features that are not relevant to the category. The 2 features selected in the first step of random initialization are used as the initial population.

$$
\begin{aligned}
F\left(X_{n+1} \mid \Omega_{n}\right) & \neq F\left(X_{n+1} \mid \Omega_{n}-Y_{n}\right), \\
x^{(i)} & =\sum_{i<j} o^{(i, j)}\left(x^{(i)}\right)(3) .
\end{aligned}
$$




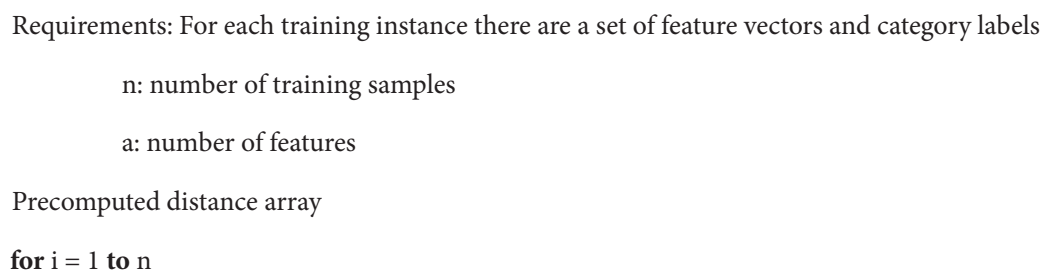

\#According to Tneari to identify neighbors and find hits and misses

Initialize hit and miss counters $h=0.0$ and $\mathrm{m}=0.0$

for $j=1$ to $n$

if $R i$ and $R j$ distance value < Tneari (use distance array judgment) then

if $R j$ is hit then

$h=h+1$ and mark this instance as hit $\}$

else if $R j$ is miss then

$\mathrm{m}=\mathrm{m}+1$ and mark this instance as miss\}

end if

end if

end for

\#Update feature weight vector W[A]

for all hits and misses

for $A=1$ to $a$

$\mathrm{W}[\mathrm{A}]=\mathrm{W}[\mathrm{A}]-\operatorname{diff}(\mathrm{A}, \mathrm{Ri}, \mathrm{H}) /\left(\mathrm{n}^{*} \mathrm{~h}\right)+\operatorname{diff}(\mathrm{A}, \mathrm{Ri}, \mathrm{M}) /\left(\mathrm{n}^{*} \mathrm{~m}\right)$

end for

end for

end for

Return feature score vector $\mathrm{W}$ the quality of the price feature

FIgURE 2: MultiSURF algorithm pseudocode flow display diagram.

TABLE 1: Eight features selected using the MultiSURF feature selection algorithm.

\begin{tabular}{l}
\hline Feature name \\
\hline Downlink_avg_acketsize \\
Downlink_acketsize_ar \\
Downlink_packetsize_pdf_entropy \\
Downlink_rate_packet \\
Downlinkes_rate_bytes \\
Uplink_interval_max \\
Datalink-avg-packetsize \\
Datalink_acketsize_var
\end{tabular}

Calculate the fitness of an individual, that is, first decode the individual, and then use the reduced sample set to calculate the fitness. According to fitness value selection, crossover and mutation population can be calculated using the following equation:

$$
\nabla_{\alpha}=\frac{w-\varepsilon \nabla_{w}(w, \alpha)}{L\left(\left(w^{*}\right)\right.} .
$$

We add all the new individuals from the previous step to the population to form a new population.

\section{Applicability and Comparative Test of Cultural IP Model}

In this chapter, we will conduct practical and comparative tests on the local cultural IP development and cultural and creative design models based on the Internet of Things and data mining established above. The test will further verify that the improved MultiSURF combined with the genetic algorithm can have a more stable and applicable performance. We will mainly analyze the scalability and accuracy 


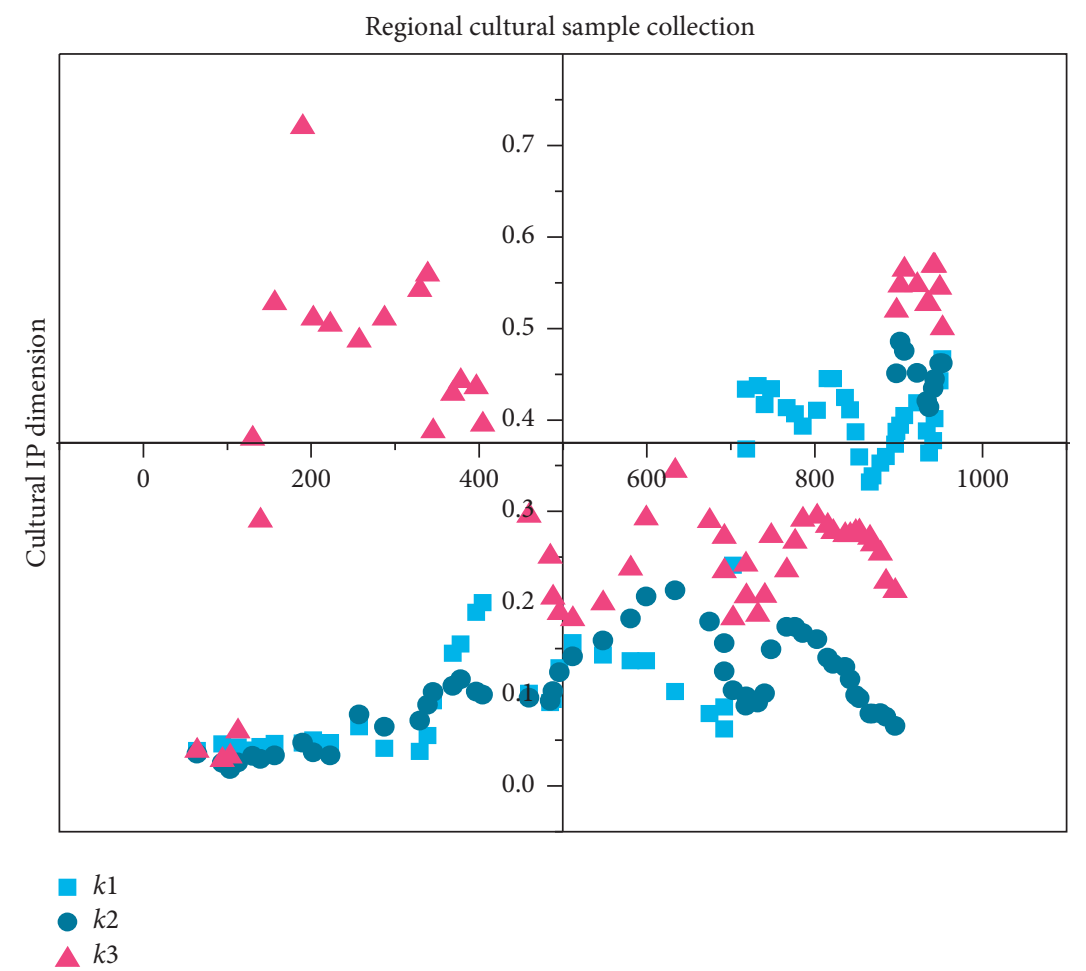

FIGURE 3: Clustering results of the MultiSURF algorithm under different $k$ values.

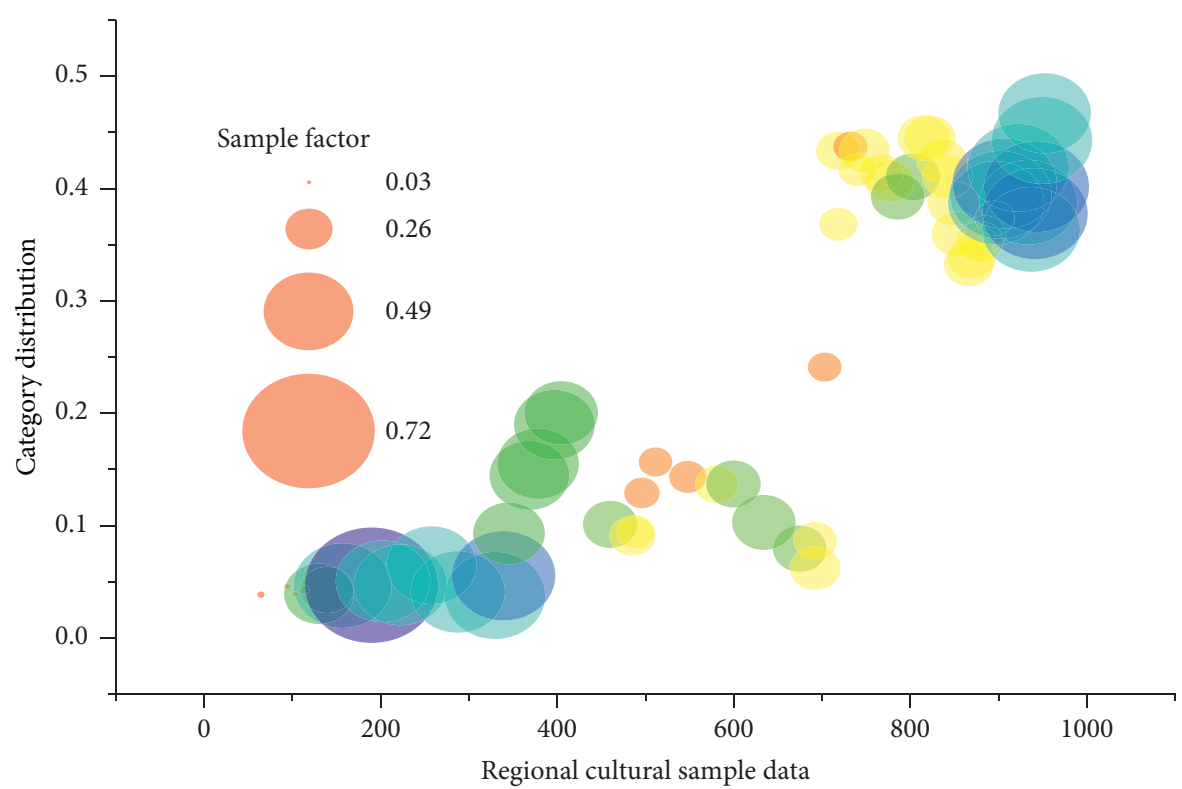

FIgURE 4: The influence of sample factors on local cultural IP development algorithm.

of the algorithm to verify whether the improved algorithm has good robustness under the Spark cluster.

4.1. Local Cultural IP Development Data Sources. The benchmark dataset used in the experiment in this section is the scoring dataset provided by the Web scope project team. In this section, the experiment extracts a subdataset containing nearly 20,000 scoring records from the scoring dataset, according to the principle of random sampling, and selects 15,000 cultural data as the test set, and its data size is the same as another score provided by the project team. The datasets are comparable.

4.2. Local Cultural IP Development Model Test. The collected data are first classified by the MultiSURF algorithm, and the final clustering result is 9 . The classification description of 


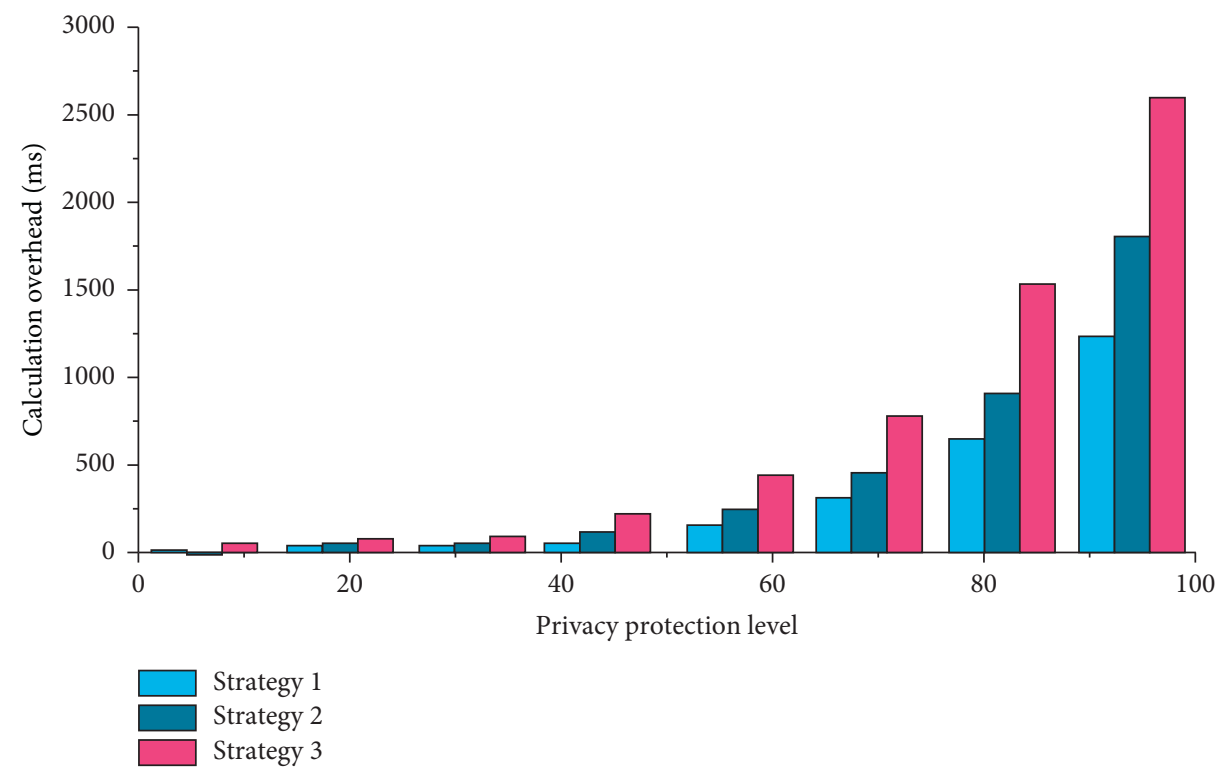

FIgURE 5: Model performance of the local cultural IP development model under different $k$ values.

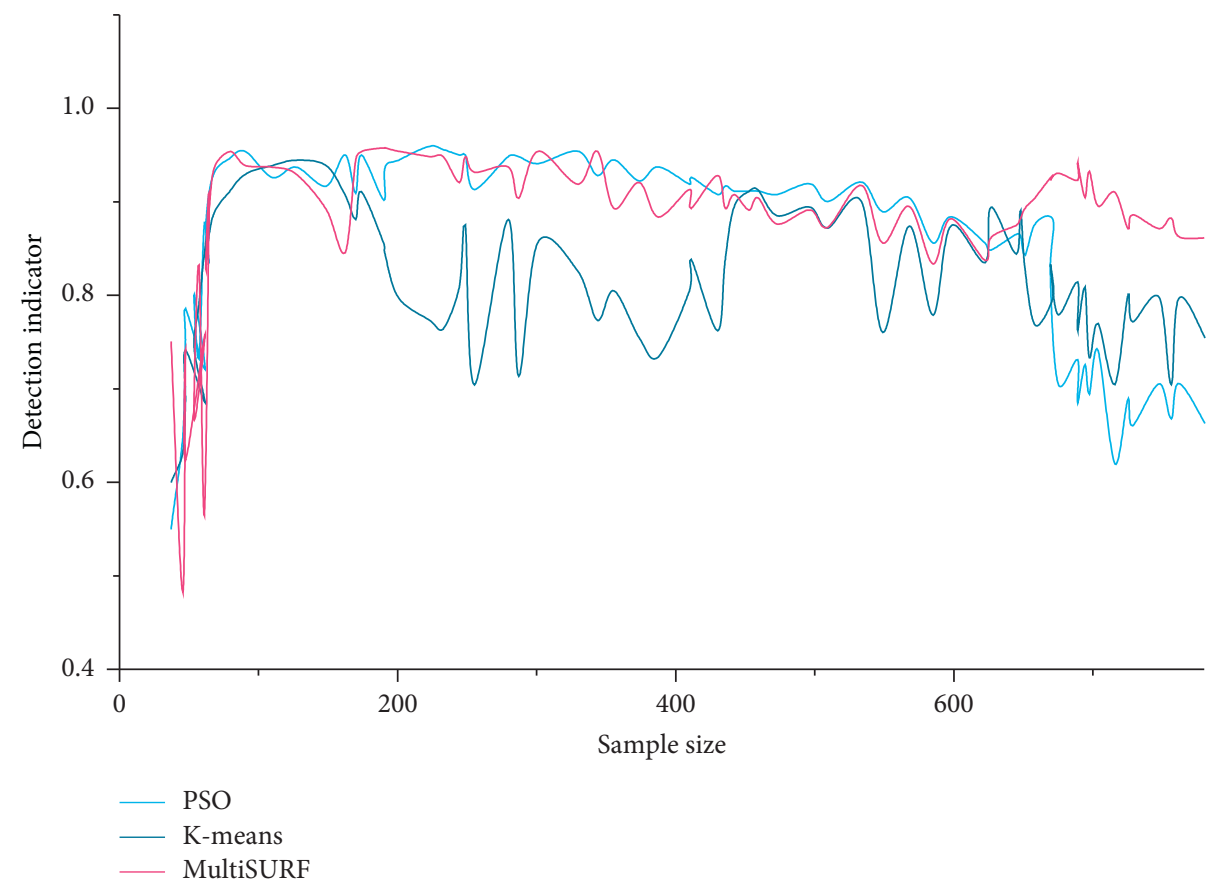

FIGURE 6: Accuracy analysis of local cultural IP development models with different algorithms.

this algorithm can be divided into 9 categories, namely, geographical resources, ancient texts, historical relics, ethnic education, humanities and economics, social customs, ideology and religion, culture and art, regional news, and other categories. 9 is the clustering center $k$ of the $K$-means algorithm, and the manually classified data are also 9 categories, indicating that the MultiSURF algorithm classification result is correct. The clustering results of the MultiSURF algorithm under different $k$ values are shown in Figure 3.
For the final clustering results, it can be seen from Figure 3 that the directions of historical cultural relics, social customs, and so on account for a relatively large proportion, and the abovementioned directions are considered to have the potential and value of cultural IP development. In the economic category, there are documents in the category of historical relics. The influence of sample factors on the local cultural IP development algorithm is shown in Figure 4.

When clustering datasets of the same size, as the number of points increases, the time consumed by the algorithm 
gradually decreases. Then, perform $K$-means algorithm clustering, input the cluster center value as 9 , as the initial cluster center, and obtain the final clustering result. Because the MultiSURF algorithm determines the clustering center, there is no need to repeat iterations, and the time consumed decreases faster. At the same time, as the number of nodes increases, the workload of a single node is reduced, making the overall clustering time less and less. The model performance of the local cultural IP development model under different $k$ values is shown in Figure 5.

The larger the expansion ratio is, the more advantageous the parallel algorithm is. The speedup comparison result of the MultiSURF algorithm, $K$-means, and PSO algorithm is shown in Figure 6.

It can be seen from Figure 6 that as the number of nodes in the cluster increases, the utilization of the cluster becomes higher and higher, but the MultiSURF algorithm increases faster than the $K$-means algorithm, indicating that the improved algorithm is suitable for a distributed environment. Speedup is the ratio of the time required for a single node to the time required for a cluster of $r$ nodes. However, due to the limitation of nodes, the speedup ratio cannot be increased without limit. The expansion ratio can test the efficiency of algorithm parallelization. Figure 6 describes the change trend of the expansion ratio of the $K$-means algorithm and the MultiSURF algorithm under the Data2 dataset as the number of nodes increases. In the same dataset and cluster environment, the expansion ratio of the $K$-means algorithm and the MultiSURF algorithm has the same trend. The difference is that the MultiSURF algorithm is faster than the $K$-means algorithm and approaches a certain fixed value, which shows that the MultiSURF based on Spark algorithm has better scalability.

\section{Conclusion}

Since the beginning of 21st century, "promoting tourism with culture, commemorating culture with tourism, and integrating culture and tourism" has become an important trend in the development of global cultural tourism industry. This paper combines stakeholder theory along with new public service theory and other related theories, using literature research, field investigation, case analysis, and other research methods to analyze the development of different types of cultural tourism resources before and after the disaster. Limited by the level of personal research ability and objective reality, this paper still has some areas to be improved. First, the literature review is not comprehensive and in-depth, and the materials and data used in the paper may have lags and limitations. Future research may further tend to integrate the above research fields to varying degrees. We will comprehensively use economics, tourism, cultural studies, management, sociology, and other multidisciplinary research perspectives and methods, combined with a specific research object for related research.

\section{Data Availability}

The datasets used and/or analyzed during the current study are available from the corresponding author on reasonable request.

\section{Conflicts of Interest}

The authors declare that they have no conflicts of interest.

\section{References}

[1] S. Zhang, X. Xu, J. Peng, K. Huang, and Z. Li, "Physical layer security in massive internet of things: delay and security analysis," IET Communications, vol. 13, no. 1, pp. 93-98, 2019.

[2] X. U. Yi, "Research on security and reliability of smart home system based on internet of things," Environmental Technology, vol. 12, no. 3, pp. 324-341, 2019.

[3] W. Xu, C. Varghese, I. P. Bissett, G. O'Grady, and C. I. Wells, "Network meta-analysis of local and regional analgesia following colorectal resection," British Journal of Surgery, vol. 107, no. 2, pp. e109-e122, 2020.

[4] S. Yumin, "Research and simulation of large data differentiation classification technology under the internet of things," in Proceedings of the 2018 International Conference on Intelligent Transportation, Big Data \& Smart City (ICITBS), Xiamen, China, January 2018.

[5] C. K. Wu, K. F. Tsang, Y. Liu, H. Zhu, H. Wang, and Y. Wei, "Critical internet of things: an interworking solution to improve service reliability," IEEE Communications Magazine, vol. 58, no. 1, pp. 74-79, 2020.

[6] S. Venkatraman, B. Surendiran, and P. Arun Raj Kumar, "Spam e-mail classification for the Internet of Things environment using semantic similarity approach," The Journal of Supercomputing, vol. 76, no. 1, pp. 756-776, 2020.

[7] C. N. Songer, G. S. Calip, N. Srinivasan, V. Maziero Barbosa, and J. T. Pham, "Factors influencing antibiotic duration in culture-negative neonatal early-onset sepsis," Pharmacotherapy, vol. 41, no. 1, 2021.

[8] X. Yu, Q. Hu, H. Li, J. Du, J. Gao, and L. Sun, "Cross-domain recommendation based on latent factor alignment," Neural Computing and Applications, vol. 7, no. 2, pp. 215-230, 2021.

[9] J. Shu, M. Deng, and J. He, "Optimization of ship-borne anticollision sounding system based on internet of things and full convolution neural network," Journal of Coastal Research, vol. 103, pp. 757-761, 2020.

[10] M. D. Rizzo, R. B. Crawford, A. Bach, S. Sermet, A. Amalfitano, and N. E. Kaminski, "Imiquimod and interferon-alpha augment monocyte-mediated astrocyte secretion of MCP-1, IL-6 and IP-10 in a human co-culture system," Journal of Neuroimmunology, vol. 333, Article ID 576969, 2019.

[11] V. Radonjic, M. S. Dogatovic, M. J. Stanojevic, and N. Mladenovic, "Revenue maximization of Internet of things provider using variable neighbourhood search," Journal of Global Optimization, vol. 78, 2020.

[12] C. E. Morgan and H. Donald, "IP247: the on-service vascular surgery practice management strategy maintains local productivity while allowing expansion of outreach practice," Journal of Vascular Surgery, vol. 67, no. 6, p. e151, 2018.

[13] M. Mayer and A. J. Baeumner, "A megatrend challenging analytical chemistry: biosensor and chemosensor concepts ready for the internet of things," Chemical Reviews, vol. 119, no. 13, 2019.

[14] C. Massip, H. Guet-Revillet, M. Grare, A. Sommet, and D. Dubois, "Enhanced culture recovery of campylobacter with modified cary-blair medium: a practical field experience," Journal of Microbiological Methods, vol. 149, pp. 53-54, 2018.

[15] I. P. Massad, M. Avila, L. Contreras-Porcia, and C. Bulboa Contador, "Spores re-suspending technology, a new system 
improving spore seeding for culture of commercial red seaweeds," Aquaculture, vol. 526, 2020.

[16] M. A. Jan, F. Khan, M. Alam, and M. Usman, "A payloadbased mutual authentication scheme for internet of things," Future Generation Computer Systems, vol. 92, pp. 1028-1039, 2019.

[17] M. Khanipour, K. Niknami, and M. Abe, "Challenges of the fars neolithic chronology: an appraisal," Radiocarbon, vol. 63, no. 2, pp. 693-712, 2020.

[18] M. Ke, Z. Gao, Y. Wu, X. Gao, and K. K. Wong, "Massive access in cell-free massive MIMO-based internet of things: cloud computing and edge computing paradigms," IEEE Journal on Selected Areas in Communications, vol. 39, no. 3, pp. 756-772, 2021.

[19] F. Ip, G. Lewis, and C. Lefevre, "Author accepted manuscript: carotenoid skin coloration enhances face and body attractiveness: a cross-cultural study," Quarterly Journal of Experimental Psychology, vol. 72, no. 2, 2019.

[20] Y. Hasegawa, R. Ishii, Y. Kyutoku, . Dan, and B. Rousseau, "Biases in paired preference tests: cross-cultural comparison of Japanese and American consumers," Journal of Sensory Studies, vol. 34, no. 3, 2019.

[21] X. Yu, F. Jiang, J. Du, and D. Gong, “A cross-domain collaborative filtering algorithm with expanding user and item features via the latent factor space of auxiliary domains," Pattern Recognition, vol. 94, pp. 96-109, 2019.

[22] E. Hittinger and P. Jaramillo, "Internet of things: energy boon or bane?” Science, vol. 364, no. 6438, pp. 326-328, 2019.

[23] B. Comery, W. R. G. Perry, S. Young et al., "Delivery of surgical care in Samoa: perspectives on capacity, barriers and opportunities by local providers," Anz Journal of Surgery, vol. 90, no. 10, pp. 1910-1914, 2020.

[24] W.-C. Chen, J.-S. Niu, I.-P. Liu et al., "Study of a palladium (Pd)/aluminum-doped zinc oxide (AZO) hydrogen sensor and the kalman algorithm for internet-of-things (IoT) application," IEEE Transactions on Electron Devices, vol. 67, no. 10, pp. 4405-4412, 2020.

[25] J. Chauhan and P. Goswami, "An integrated metaheuristic technique based energy aware clustering protocol for internet of things based smart classroom," Modern Physics Letters B, vol. 34, no. 22, Article ID 2050360, 2020.

[26] R. Ceipek, J. Hautz, A. De Massis, K. Matzler, and L. Ardito, "Digital transformation through exploratory and exploitative internet of things innovations: the impact of family management and technological diversification," Journal of Product Innovation Management, vol. 38, no. 1, pp. 142-165, 2021.

[27] C. Landa-Solís, A. Olivos, B. Olivos-Díaz et al., "Behavior of multipotent stem cells isolated in mobilized peripheral blood from sheep after culture with human chondrogenic medium," Tissue \& Cell, vol. 52, pp. 116-123, 2018.

[28] X. Yu, Y. Chu, F. Jiang, Y. Guo, and D. Gong, "SVMs classification based two-side cross domain collaborative filtering by inferring intrinsic user and item features," KnowledgeBased Systems, vol. 141, pp. 80-91, 2018.

[29] A. Villa-Henriksen, G. T. C. Edwards, L. A. Pesonen, O. Green, and C. Aage, "Internet of Things in arable farming: implementation, applications, challenges and potential," Biosystems Engineering, vol. 191, pp. 60-84, 2020.

[30] H. A. A. Al-Kashoash, H. Kharrufa, Y. Al-Nidawi, and A. H. Kemp, "Congestion control in wireless sensor and 6LoWPAN networks: toward the internet of things," Wireless Networks, vol. 25, no. 8, pp. 4493-4522, 2019.
[31] X. Yu, J. Yang, and Z. Xie, "Training SVMs on a bound vectors set based on fisher projection," Frontiers of Computer Science, vol. 8, no. 5, pp. 793-806, 2014.

[32] G. Royer, F. Fourreau, B. Boulanger et al., "Local outbreak of extended-spectrum $\beta$-lactamase SHV2a-producing Pseudomonas aeruginosa reveals the emergence of a new specific sublineage of the international ST235 high-risk clone," Journal of Hospital Infection, vol. 104, no. 1, pp. 33-39, 2020. 